\title{
Le programme Namaste, une psychothérapie de groupe basée sur le yoga pour les jeunes ayant un vécu de traumas complexes: une série d'étude de cas \\ Rosée BRUNEAU-BHERER ${ }^{1}$, Sara TREMBLAY ${ }^{1}$, Alexandra MATTE-LANDRY², Camille PÉPIN ${ }^{3}$ et Delphine COLLIN-VÉZINA²
}

\footnotetext{
Centre intégré universitaire en santé et service sociaux de la Capitale-Nationale (CIUSSS-CN)

2 School of Social Work, McGill University

3 Université Laval
}

Correspondance : Rosée Bruneau-Bherer, Centre intégré universitaire en santé et service sociaux de la Capitale-Nationale (CIUSSS-CN), 3510 rue Cambronne, Québec, Québec, Canada G1E 7H2.

Courriel : rosee.bruneau-bherer.ciussscn@ssss.gouv.qc.ca

\section{Résumé}

Introduction : Les enfants qui ont un vécu des traumas complexes sont à risque de développer de graves difficultés d'attachement, d'autorégulation et de comportement. Des études exploratoires démontrent que la pratique du yoga permet d'améliorer leur fonctionnement et diminuer leurs symptômes d'internalisation et d'externalisation.

Objectifs : Le programme Namaste, une adaptation du manuel «Yoga-Based Psychotherapy » (Beltran et al. 2014) vise l'amélioration de l'autorégulation et de la santé mentale. Cette étude a pour objectif de documenter ses effets chez les enfants qui ont vécu des traumas complexes.

Méthode : Douze séances ont été animées auprès d'enfants âgés de 6 à 13 ans suivis par un centre de protection de l'enfance. Les séances combinaient des postures de yoga, des exercices de respiration, et d'autres activités favorisant l'autorégulation et la socialisation. Le fonctionnement et les symptômes des enfants ont été mesurés avec le questionnaire BASC-3 avant et après l'intervention. Deux études de cas détaillées décrivent les impacts potentiels de cette intervention.

Résultats: Les deux études de cas démontrent des impacts positifs sur les symptômes d'internalisation et d'externalisation ainsi que l'autorégulation, mais aucun effet sur l'estime de soi et les compétences sociales.

Implications : Le programme Namaste pourrait être une intervention prometteuse pour améliorer le fonctionnement et réduire les symptômes psychologiques chez cette population. L'intégration des principes du yoga sensible aux traumas, la modalité de groupe et l'accent mis sur l'autorégulation semblent des éléments-clés. D'autres études de plus grande envergure sont nécessaires pour confirmer les impacts positifs potentiels de cette intervention.

Mots-clés : Yoga sensible au trauma; Trauma complexe; Psychothérapie de groupe. 
Le programme Namaste, une psychothérapie de groupe basée sur le yoga pour les jeunes

ayant un vécu de traumas complexes : une série d'étude de cas

\section{Introduction}

Les enfants et adolescents qui reçoivent des services en protection de la jeunesse sont à risque de présenter des difficultés psychologiques en lien avec leur vécu familial, souvent traumatique (p. ex., expériences d'abus physique, psychologique ou sexuel, exposition à de la violence familiale, séparation des parents, présence d'un trouble mental chez un membre de la famille). Le terme trauma complexe englobe le fait d'avoir vécu des traumas interpersonnels, chroniques et répétés, ainsi que les séquelles complexes et durables qui leur sont associées (Milot et al., 2018, p. 10). Les séquelles incluent notamment des symptômes de trouble de stress post-traumatique (TSPT), ainsi que des déficits dans plusieurs sphères du développement (Godbout et al., 2018). Par exemple, les jeunes peuvent avoir des difficultés relationnelles, identitaires, d'attachement, de régulation des émotions et de mentalisation, des problèmes cognitifs et d'apprentissage ou des comportements dysfonctionnels (Godbout et al., 2018). Les séquelles comprennent aussi un ensemble de symptômes psychologiques d'externalisation (p. ex., crise agressive, opposition, agitation et provocation) et d'internalisation (p. ex., dépression, anxiété, retrait social et somatisation). Ces problèmes peuvent perdurer à l'âge adulte (Cloitre et al., 2009; van der Kolk, 2014) et requièrent souvent des soins psychologiques spécialisés. Parfois, un placement en dehors du milieu familial (p. ex., centre de réadaptation, foyer de groupe ou famille d'accueil) est l'option qui répond le mieux aux besoins de sécurité et de développement de l'enfant. Au Québec, Collin-Vézina et ses collaborateurs (2011) ont rapporté que $83 \%$ des jeunes hébergés en centre de réadaptation avaient vécu au moins une forme de maltraitance et que 76 \% d'entre eux avaient été victimes de formes multiples de mauvais traitements. La prévalence extrêmement élevée chez cette clientèle des traumatismes relationnels, et des séquelles associées, nécessite l'implantation d'interventions sensibles aux traumas. Les approches thérapeutiques qui visent à intégrer le corps et l'esprit (« mind-body interventions ») sont de plus en plus utilisées dans le traitement des traumas complexes (Mayer, 2019). Une de ces approches, le yoga sensible aux traumas, est une avenue intéressante pour rejoindre cette clientèle puisque la régulation des émotions constitue une pierre angulaire du traitement (Blaustein \& Kinniburgh, 2018; Spinazzola et al., 2001). L'objectif de cette étude est de décrire les impacts potentiels de la participation au Programme Namaste, une psychothérapie basée sur le yoga, chez des enfants ayant un vécu de traumatismes complexes.

\section{Le yoga sensible aux traumas}

La pratique du yoga sensible aux traumas invite, par le mouvement et la respiration, à observer les sensations du corps et à passer à l'action pour améliorer son bien-être (p. ex., maintenir la posture, l'approfondir ou la cesser; Emerson et al. 2009). Il peut s'agir de l'amorce d'un processus thérapeutique important.

Plusieurs avantages rendent l'approche de la psychothérapie par le yoga intéressante, notamment le risque minimal encouru, la bonne acceptabilité sociale, la facilité d'adaptation à diverses populations et contextes ainsi que les avantages sur la santé associés à la pratique d'une activité physique (Sciarrino et al., 2017). II semble aussi que le rapport coût-bénéfice soit avantageux (Macy et al., 2018). De plus, il a été démontré que le yoga a une variété d'effets thérapeutiques sur la santé psychologique et cognitive, notamment l'amélioration de la régulation émotionnelle (Macy et al., 2018), qui est fréquemment affectée chez les victimes de traumatismes complexes.

Des lignes directrices ont été développées pour encadrer la pratique du yoga auprès de personnes ayant vécu des traumas (Emerson et al., 2009). Premièrement, l'environnement physique doit favoriser un sentiment de confiance et de sécurité. Par exemple, l'absence de fenêtres par lesquelles on pourrait observer l'intérieur, l'insonorisation et l'absence de miroirs peuvent favoriser la relaxation. Deuxièmement, la manière d'animer les postures de yoga doit être adaptée; les consignes sont formulées comme des suggestions et l'accent est mis sur le respect des désirs et des limites de chacun. Aussi, les participants sont encouragés à être à l'écoute de leur corps et de leurs émotions; si une posture est inconfortable pour une personne, peu importe la raison, elle doit se respecter. La personne peut alors modifier la posture, sa durée ou encore rester debout ou assise pendant un certain temps. Troisièmement, les qualités des intervenants qui animent les séances sont importantes; une bonne connaissance des impacts des traumas sur le développement et des réactions physiologiques associées est essentielle. En plus d'une bonne connaissance du yoga, une attitude d'ouverture et de non-jugement favorise le bon déroulement. Une attention particulière au langage utilisé lors de l'animation est de mise pour éviter de déclencher des réactions post-traumatiques. Le vocabulaire pour décrire les postures est simplifié afin de respecter les limites langagières parfois présentes. Quatrièmement, ces auteurs proposent d'éviter les contacts physiques; les corrections verbales étant plus appropriées. L'un des mécanismes d'action proposés pour expliquer l'efficacité du yoga chez les victimes de traumatismes est la régulation du système nerveux autonome. Ce système se compose de deux voies qui travaillent de façon antagoniste. La voie sympathique 
prépare le corps à avoir une réponse adaptée face à un stresseur (van der Kolk, 2014). La voie parasympathique assure la conservation de l'énergie en ralentissant les fonctions de l'organisme (Bear et al., 2007). Chez les enfants ayant vécu des traumas, le système sympathique devient programmé en mode survie de façon permanente. Ainsi, il s'active, et ce, même dans un contexte sécuritaire (Spinazzola et al., 2011). Le yoga permet d'améliorer la résilience au stress en favorisant l'équilibre entre la suractivation du système nerveux sympathique et la réponse du système nerveux parasympathique, nécessaire à l'apaisement (Ross \& Thomas, 2010).

\section{L'efficacité des interventions utilisant le yoga chez les victimes de traumas}

Les résultats d'une méta-analyse conduite sur 24 études contrôlées randomisées indiquent que les interventions basées sur la pleine conscience, tel le yoga, sont efficaces dans la réduction des symptômes liés aux traumatismes psychologiques chez les adultes (Taylor et al., 2020). Les interventions durant plus de huit semaines seraient particulièrement efficaces (Taylor et al., 2020). Dans une recension exhaustive, Macy et ses collaborateurs (2018) ont analysé 13 revues de la littérature qui démontrent que la pratique du yoga est efficace chez l'adulte en tant que traitement des impacts liés à divers types de traumatismes (p. ex., le TSPT, l'anxiété et la dépression). Une autre recension de la littérature portant spécifiquement sur l'efficacité des interventions de type yoga sensible aux traumas chez les femmes ayant vécu un trauma interpersonnel et qui présentent un TSPT corrobore ces conclusions, particulièrement chez une population qui ne répond pas aux thérapies cognitives-comportementales traditionnelles (Nolan, 2016). Une étude récente ayant utilisé un devis non randomisé chez neuf femmes ayant un TSPT chronique et résistant au traitement supporte également l'efficacité de ce type d'intervention à réduire significativement les symptômes liés au TSPT et dissociatifs, davantage que des traitements similaires administrés sur une plus courte période (Price et al., 2017). Cette étude précise par ailleurs que la durée de l'intervention et l'ajout d'une pratique à la maison ont un impact positif. Le nombre d'études portant sur les effets du yoga sur la santé mentale est en croissance et leurs résultats sont prometteurs. Actuellement, les appuis empiriques sont insuffisants pour recommander le yoga sensible aux traumas comme traitement principal des traumas complexes, mais sa pratique donnerait des résultats intéressants en combinaison avec d'autres thérapies plus traditionnelles (ex., psychothérapie, pharmacothérapie) (Macy et al., 2018). Aucune recension de la littérature ne s'est encore spécifiquement intéressée à cette modalité chez les jeunes enfants et adolescents victimes de traumas complexes.

L'efficacité thérapeutique du yoga en complément avec d'autres approches a cependant été démontrée chez les enfants et adolescents affectés par d'autres problématiques physiques (p. ex., difficultés cardiorespiratoires) et mentales (p. ex., difficultés attentionnelles et anxiété (Kaley-Isley et al., 2010; Galantino et al., 2008). L'étude d'Hariprasad et ses collaborateurs (2013) corrobore que l'enseignement du yoga chez les enfants pourrait avoir un impact positif sur les symptômes qui caractérisent le trouble déficitaire de l'attention avec hyperactivité (TDAH). La pratique du yoga sensible aux traumas a aussi été étudiée chez de jeunes adolescents placés hors de leur milieu familial et présentant des difficultés comportementales et émotionnelles sévères (Spinazzola et al., 2011). Les deux études de cas décrites par ces auteurs (c.-à-d., une fille âgée de 16 ans et un garçon âgé de 17 ans) font état de changements positifs chez les participants, dont l'amélioration des interactions sociales, la concentration et le contrôle des impulsions (Spinazzola et al., 2011).

Récemment, Beltran et ses collaborateurs $(2014,2016)$ ont développé un traitement psychologique de groupe basé sur le yoga. Ils ont examiné les effets d'une intervention de 14 semaines basée sur le yoga et la pleine conscience chez 10 garçons de $4^{\mathrm{e}}$ et $5^{\mathrm{e}}$ année du primaire ayant été exposés à des expériences traumatiques en milieu urbain (Beltran et al., 2016). Cette intervention visait à améliorer le fonctionnement comportemental et émotionnel de ces garçons en utilisant les éléments de base du yoga afin de promouvoir la réflexion sur soi et l'autorégulation des fonctions affectives, somatiques, comportementales et cognitives, tout en offrant des opportunités d'apprentissages relationnels. Beltran et ses collègues (2016) ont observé une diminution significative des pensées intrusives, de l'activation émotionnelle, et de la rumination à la suite de l'intervention. Toutefois, la généralisation des résultats est limitée en raison de la taille de l'échantillon et d'autres études similaires sont nécessaires pour mieux comprendre les effets de la psychothérapie basée sur le yoga chez les jeunes victimes de traumas.

\section{Objectifs du programme Namaste}

Le programme Namaste a été traduit et adapté par deux psychologues d'un centre de protection de l'enfance au Québec (Canada) à partir du programme «Yoga based psychotherapy: a group protocol for children » développé par Beltran et ses collaborateurs $(2014,2016)$ avec l'autorisation des auteurs. Le programme Namaste s'adresse aux 
enfants âgés de 6 à 12 ans ayant un vécu de traumatismes complexes et vise l'amélioration du fonctionnement psychologique par le biais de ces concepts clés:

- La sécurité et les frontières personnelles: enseigner à l'enfant l'importance de respecter les limites des autres et de faire respecter les siennes, ainsi qu'à identifier les comportements et situations sécuritaires;

- La conscience de soi: développer la capacité à prendre conscience de son corps et de ses sensations par le yoga et, éventuellement, à prendre conscience de ses pensées et de ses émotions;

- L'autoapaisement: enseigner des techniques de respiration et de mouvement qui peuvent aider à se calmer;

- L'autorégulation: augmenter la durée de l'attention et les comportements dirigés vers un but et diminuer les crises;

- Les compétences: augmenter les habiletés de socialisation, de communication des besoins et des émotions, et la capacité à gérer le stress;

- L'estime de soi: développer un regard sur soi positif à travers des accomplissements et la notion d'identité propre.

Grâce à ces cibles d'intervention, il est attendu que la participation au programme influence positivement la santé mentale et le fonctionnement des jeunes participants. Cette étude pilote avait pour objectif d'explorer l'impact de la participation au programme Namaste sur le fonctionnement, les symptômes d'internalisation et d'externalisation, ainsi que l'autorégulation chez des enfants suivis par les services de protection de l'enfance.

\section{Méthode}

\section{Participants}

Le programme s'adressait aux jeunes hébergés dans les foyers de groupe d'un centre de protection de l'enfance qui désiraient apprendre à mieux gérer leurs émotions par le mouvement. Les éducateurs ont référé les jeunes intéressés. Les jeunes présentant une problématique suicidaire aiguë, alimentaire, de violence ou de sommeil ou encore toute autre condition de santé jugée contrindiquée n'ont pas pu participer au groupe. Deux groupes de sept jeunes, âgés de 6 à 13 ans ont participé au programme Namaste. Ces garçons $(n=11)$ et ces filles $(n=3)$ présentaient des difficultés de régulation des émotions selon leurs intervenants. Des évaluations pré et post-intervention ont été effectuées avec le deuxième groupe ( $n=7$; quatre garçons et trois filles). De ces sept participants, un garçon n'a pas pu compléter le programme en raison d'un changement de milieu de vie et une fille n'a assisté qu'à huit séances sur 12. Parmi les cinq autres jeunes, les parents de deux d'entre eux ont pu être joints et ont consenti à ce que les données cliniques recueillies dans le cadre de ce service soient utilisées à des fins de recherche. Les jeunes ont aussi donné leur assentiment. Ce projet de recherche a été approuvé par le comité d'éthique à la recherche du centre de protection de l'enfance (2020-1917). Considérant le nombre limité de participants $(n=2)$, l'étude de cas a été privilégiée comme méthodologie de recherche à l'instar de Spinazzola et ses collaborateurs (2011).

\section{L'intervention}

Le manuel original (Beltran et al., 2014, 2016) comprenait 14 séances de 90 minutes. La première et la 14e séance s'adressaient aux parents des enfants participants et n'ont pas été incluses dans ce projet puisque les jeunes participants étaient hébergés en dehors de leur milieu familial. Les 12 autres séances ont été traduites et adaptées. Les séances incluaient toujours une séance de yoga (20 à $40 \mathrm{~min}$ ), une activité psychoéducative (15 à $25 \mathrm{~min}$ ), des jeux favorisant l'intégration des cibles d'intervention (15 à $30 \mathrm{~min}$ ) et du temps consacré aux routines du groupe (p. ex., accueil, collations, auto-évaluation, rangement; environ $15 \mathrm{~min}$ ). Le Tableau 1 présente les thèmes spécifiques de chaque séance. Les séances étaient animées par deux psychologues formées pour travailler avec les enfants ayant vécu des traumas complexes, dont l'une est aussi professeure de yoga. 
Tableau 1. Thèmes des 12 séances du programme Namaste

\begin{tabular}{cl}
\hline Séances & \multicolumn{1}{c}{ Thèmes } \\
\hline 1 & Séance d'accueil et d'introduction au yoga \\
2 & Conscience du corps \\
3 & Focus sur la respiration \\
4 & Introduction à la relaxation \\
5 & Conscience de soi \\
6 & Identification des émotions \\
7 & Prendre soin de soi et sentiment de sécurité : frontières et \\
8 & espace personnel \\
9 & Conscience des autres \\
10 & Travail en équipe \\
11 & Consolidation de la relaxation et des stratégies d'autorégulation \\
12 & Grande fête finale du groupe \\
\hline
\end{tabular}

\section{Outils d'évaluation}

Le questionnaire Système d'évaluation du comportement de l'enfant, version pour francophones du Canada (BASC-3; Reynolds \& Kamphaus, 2015) a été utilisé pour mesurer le fonctionnement des jeunes et leur symptomatologie. Ce questionnaire est un système multidimensionnel qui évalue les différentes facettes du fonctionnement des jeunes âgés de 2 à 25 ans (Reynolds \& Kamphaus, 2015). Il permet d'évaluer les forces et les déficits en mesurant des comportements adaptés et dysfonctionnels. La validité interne (alpha de Cronbach variant entre ,82 et ,96 selon les échelles) et la fiabilité (coefficient de fidélité variant entre,78 et, 89 selon les échelles) de cet outil sont jugées excellentes (Reynolds \& Kamphaus, 2016). Dans cette étude, la version autorapportée pour les jeunes âgés de 8 ans et plus ainsi que la version pour les parents, remplie par l'éducateur qui connaissait le mieux le jeune, ont été utilisées.

Le questionnaire a été rempli au foyer de groupe juste avant le début de l'intervention (pré) et après les 12 séances (post). Les jeunes ont été accompagnés par les éducateurs selon leur niveau de lecture et de compréhension. Des changements étaient attendus sur plusieurs échelles du BASC-3 en lien avec les concepts clés ciblés par l'intervention. Les échelles Indice de symptômes comportementaux, Problèmes d'externalisation, Problèmes d'internalisation, Résilienceet Habiletés adaptatives, ont été utilisées pour évaluer le fonctionnement général et la santé mentale. Les échelles Contrôle de la colère, Maîtrise de soi émotionnelle et Fonctions exécutives ont été utilisées pour mesurer l'autorégulation et, indirectement, la conscience de soi et l'autoapaisement qui y sont étroitement liées. Les échelles Habiletés sociales, Communication fonctionnelle et Relations interpersonnelles ont été utilisées pour évaluer la sécurité et les frontières personnelles ainsi que les compétences sociales. Enfin, l'échelle Estime de soia été utilisée pour évaluer le concept du même nom. Les scores bruts ont été transformés en score $t$ $(M=50, E ́ T=10)$, permettant une comparaison avec un large échantillon selon le genre et l'âge des participants. Les scores se situant entre 40 et 60 sont considérés comme dans la norme; les scores entre 60 et 70 (pour les comportements dysfonctionnels) ou encore entre 40 et 30 (pour les comportements adaptatifs) se situent dans la zone à risque, et peuvent indiquer la présence d'une problématique. Les scores au-dessus de 70 et en dessous de 30 sont considérés comme cliniquement significatifs.

Par ailleurs, au terme des 12 séances, les éducateurs des jeunes participants ont été invités à transmettre informellement leurs commentaires et appréciations sur l'intervention (p. ex., durée, activités proposées, intérêt des jeunes, pratiques à la maison, améliorations perçues au quotidien, etc.). Ces informations ont été notées au dossier psychologique des jeunes et considérées, qualitativement, dans la présente étude. Les informations suivantes ont aussi été extraites du dossier psychologique des jeunes pour documenter leur vécu avant, pendant et après l'intervention: les données sociodémographiques (âge, genre, milieu de vie), le niveau académique, les forces et difficultés principales et la présence (ou l'absence) aux différentes séances. L'histoire de vie et les diagnostics ont été extraits des rapports d'évaluation disponibles. 


\section{Analyses}

Les notes de suivi des séances de psychothérapie des deux études de cas ont été utilisées pour décrire qualitativement les impacts de l'intervention sur la santé mentale et le fonctionnement des jeunes participants. La correction et l'interprétation des résultats au BASC-3 ont été faites à l'aide du logiciel d'analyse des résultats sur la plateforme Q-Global de Pearson. L'appréciation des résultats se base sur l'inspection graphique, le changement de catégorie (zone clinique, à risque ou normale), ainsi que le sens du changement (amélioration vs dégradation). Les études de cas d'Annie et de Jessy sont présentées selon une approche clinique et individualisée. Leur nom, ainsi que certains détails de leur vie ont été modifiés pour préserver leur anonymat, mais les résultats présentés sont réels.

\section{Résultats}

\section{Annie}

Annie est une jeune âgée de 12 ans au moment de participer au programme Namaste. Elle cumule un vécu traumatique de négligence, de maltraitance et de ruptures de lien avec ses parents, sa fratrie et d'autres personnes significatives. Elle présente des difficultés d'attachement, de santé mentale et de comportements au long cours. Annie a vécu ses quatre premières années avec sa mère, son père et une demi-sœur. Son milieu familial était extrêmement maltraitant et négligent et Annie a été retirée de ce milieu lorsque sa demi-sœur a dénoncé les abus sexuels dont elle avait été victime. Il est possible qu'Annie ait aussi été abusée, ou encore, été témoin des abus sexuels et de la violence subie par sa demi-sœur. La mère d'Annie a été violentée psychologiquement et physiquement en sa présence. Annie a été retirée d'urgence de son milieu familial et d'abord placée en famille d'accueil, puis hospitalisée en pédopsychiatrie pour une évaluation. Elle a reçu à l'âge de 5 ans plusieurs diagnostics de trouble de l'attachement, trouble déficitaire de l'attention avec hyperactivité, trouble du langage et trouble de l'humeur non spécifié. Elle a ensuite séjourné dans deux foyers de groupe dans lesquels les éducateurs utilisent une approche sensible aux traumas. Au moment de sa participation au programme, elle fréquentait une école spécialisée pour les enfants qui présentent une psychopathologie sévère. Sur le plan intellectuel, Annie se situe au niveau de la limite de la déficience intellectuelle. Elle bénéficiait aussi d'un suivi psychologique individuel en plus de sa participation au programme. Elle a participé avec entrain à 11 des 12 séances du programme Namaste et elle s'impliquait bien dans toutes les activités proposées. Annie avait de la difficulté à rester concentrée sur la tâche, mais cela ne nuisait pas au déroulement des séances. Elle acceptait et comprenait bien les consignes et donnait souvent des idées très créatives. Les résultats pré-intervention d'Annie au questionnaire BASC-3 sont présentés dans les Figures 1 et 2 . Ses résultats pré-intervention témoignent de grandes difficultés dans la majorité des sphères évaluées.

Résultats post-intervention rapportés par l'éducateur. Les résultats rapportés par l'éducateur d'Annie indiquent une meilleure adaptation sur plusieurs échelles entre les deux temps de mesure (pré et post-intervention). Bien que la plupart de ses scores restent dans la zone clinique, elle semble s'être améliorée sur l'un des concepts centraux du programme : l'autorégulation (évalué par les échelles Contrôle de la colèreet Maitrise de soi émotionnelle). Toutefois, il ne semble pas y avoir de changement sur l'échelle évaluant le Fonctionnement exécutif, aussi lié à l'autorégulation. Ensuite, il semble y avoir une amélioration sur les échelles évaluant les Problèmes d'externalisation et d'internalisation, suggérant des effets positifs du programme sur sa santé mentale. Par contre, il ne semble pas y avoir de progrès aux échelles mesurant les autres concepts ciblés par le programme, soit les compétences sociales et les facteurs de protection mesurés par les échelles Habiletés sociales, Communication fonctionnelle, Habiletés adaptatives et Résilience.

Résultats post-intervention autorapportés. Globalement, il semble qu'Annie a rapporté moins de difficultés personnelles que son éducateur en percevait et qu'elle se considérait souvent comme similaire à la moyenne des filles du même âge. Sur le plan du fonctionnement général et des symptômes d'internalisation et d'externalisation, les résultats autorapportés indiquent qu'Annie a perçu une diminution de ses difficultés sur le plan des symptômes dépressifs. De plus, pour le concept des compétences sociales, elle a perçu une amélioration de ses relations interpersonnelles. Ses résultats aux autres échelles ont varié à la suite du programme, allant parfois dans le sens d'une augmentation des difficultés, tout en restant à l'intérieur de la même zone et, le plus souvent, se situaient sous le seuil à risque. Ces variations ne sont donc pas considérées comme des changements d'une importance clinique. 
Figure 1. Résultats d'Annie aux échelles du questionnaire BASC-3 tel que rapportés par son éducateur

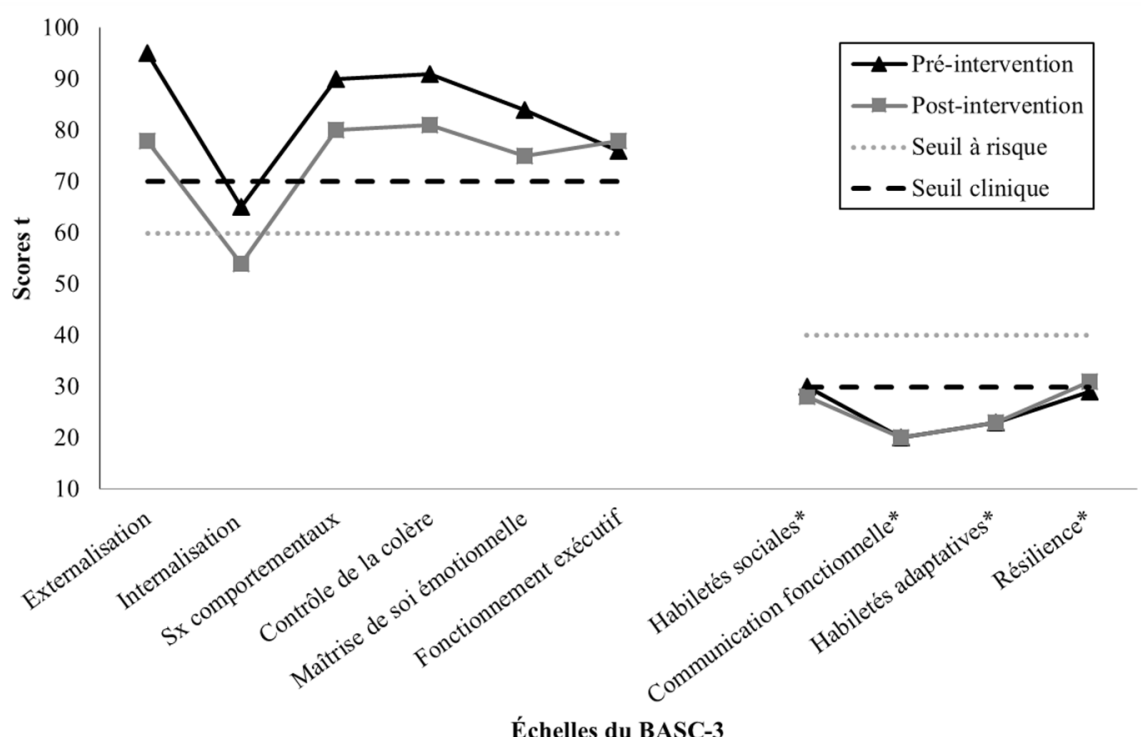

Note. Les scores des échelles identifiées avec un astérisque sont renversés

Figure 2. Résultats autorapportés par Annie aux échelles du questionnaire BASC-3

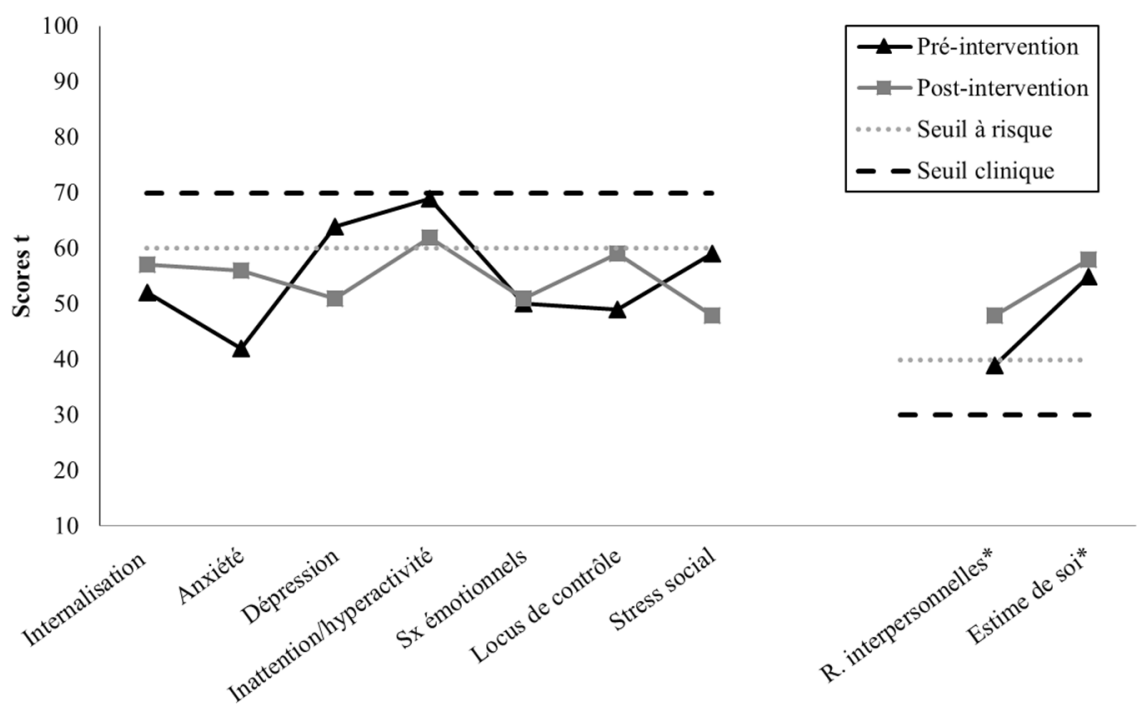

Échelles du BASC-3

Note. Les scores des échelles identifiées avec un astérisque sont renversés

Jessy

Jessy est un garçon âgé de 12 ans au moment de sa participation au programme Namaste. II a aussi vécu de nombreux changements de milieux de vie, de la négligence, des abus physiques et de mauvais traitements psychologiques au cours de son enfance. Jessy a été hébergé dans deux foyers de groupes depuis l'âge de 6 ans et il a connu deux familles d'accueil et des retours dans son milieu familial avant cela. Les deux parents de Jessy ont connu des difficultés de santé mentale, de consommation et de violence. Malgré ce vécu, Jessy est un garçon vif, agréable et capable d'entrer en relation avec les adultes et ses pairs. II fréquente une classe spécialisée pour les jeunes en difficultés 
de comportement, mais apprendrait au rythme attendu. Son dossier fait état des difficultés suivantes : trouble de l'opposition avec provocation, éléments anxieux et difficultés d'attachement. Sur le plan intellectuel, il se situerait dans la moyenne faible. Jessy était au départ réticent à participer au programme Namaste, mais grâce à l'accompagnement de son éducateur, il a rapidement pris goût aux activités proposées. II a participé à 11 séances sur 12. II était souvent un leader positif, quoiqu'il fût aussi agité et que son comportement dût parfois être recadré. À la fin du programme, il disait ne pas voir de changement chez lui et ne jamais se pratiquer au foyer, mais son entourage l'aurait observé faire discrètement certaines respirations et postures apprises dans le groupe lors de moments de stress. Les résultats pré et post-intervention de Jessy au questionnaire BASC-3 sont présentés dans les Figures 3 et 4 . Avant le programme, Jessy présentait des difficultés sur l'ensemble des échelles mesurant des comportements dysfonctionnels, mais avait aussi plusieurs facteurs de protection.

Résultats post-intervention rapportés par l'éducateur. Les résultats de Jessy, tels que rapportés par son éducateur, indiquent une amélioration sur plusieurs échelles avec des scores passant de la zone clinique à la zone à risque, ou de la zone à risque à la zone normale. En ce qui concerne le fonctionnement général, il semble y avoir eu une diminution des scores aux échelles évaluant les Problèmes d'externalisation et d'internalisation ainsi que les symptômes comportementaux entre les deux temps de mesure. De plus, en ce qui a trait au concept d'autorégulation, l'éducateur a également observé une diminution des problématiques à l'échelle Maitrise de soi émotionnelle et, dans une moindre mesure, à l'échelle Contrôle de la colère. Les facteurs de protection, comme la Résilience et les Habiletés adaptatives, se situaient dans la zone normale aux deux temps de mesures. Cependant, on observe une légère baisse des Habiletés sociales qui se retrouvaient dans la zone à risque après le programme. Le résultat à l'échelle Fonctionnement exécutifn'était pas valide dans la version pré-intervention en raison d'un trop grand nombre d'items non répondus.

Résultats post-intervention autorapportés. Tout comme Annie, Jessy s'attribuait moins de difficultés que son éducateur n'en percevait et presque toutes les échelles se situaient dans la zone normale avant sa participation au programme. À la suite de l'intervention Namaste, les résultats autorapportés de Jessy sont demeurés stables, à l'exception du Locus de contrôle qui mesure la perception de contrôle sur sa vie, qui se serait amélioré.

Figure 3. Résultats de Jessy aux échelles du questionnaire BASC-3 tel que rapportés par son éducateur

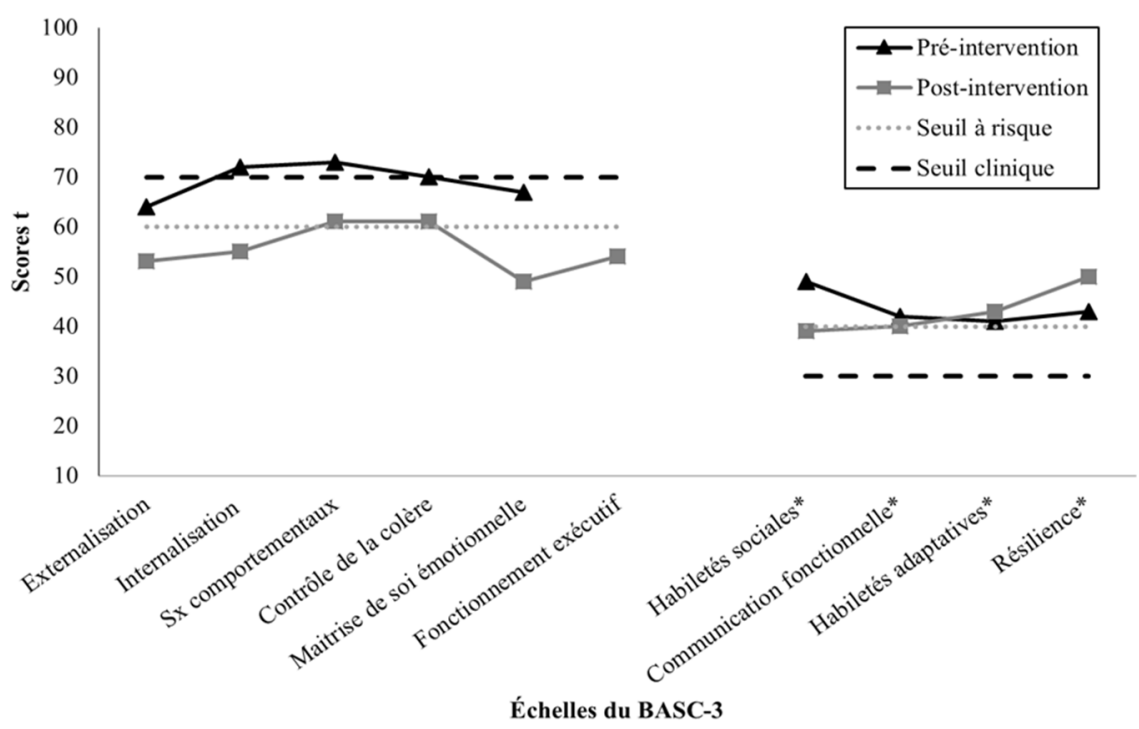

Note. Les scores des échelles identifiées avec un astérisque sont renversés 
Figure 4. Résultats autorapportés par Jessy aux échelles du questionnaire BASC-3

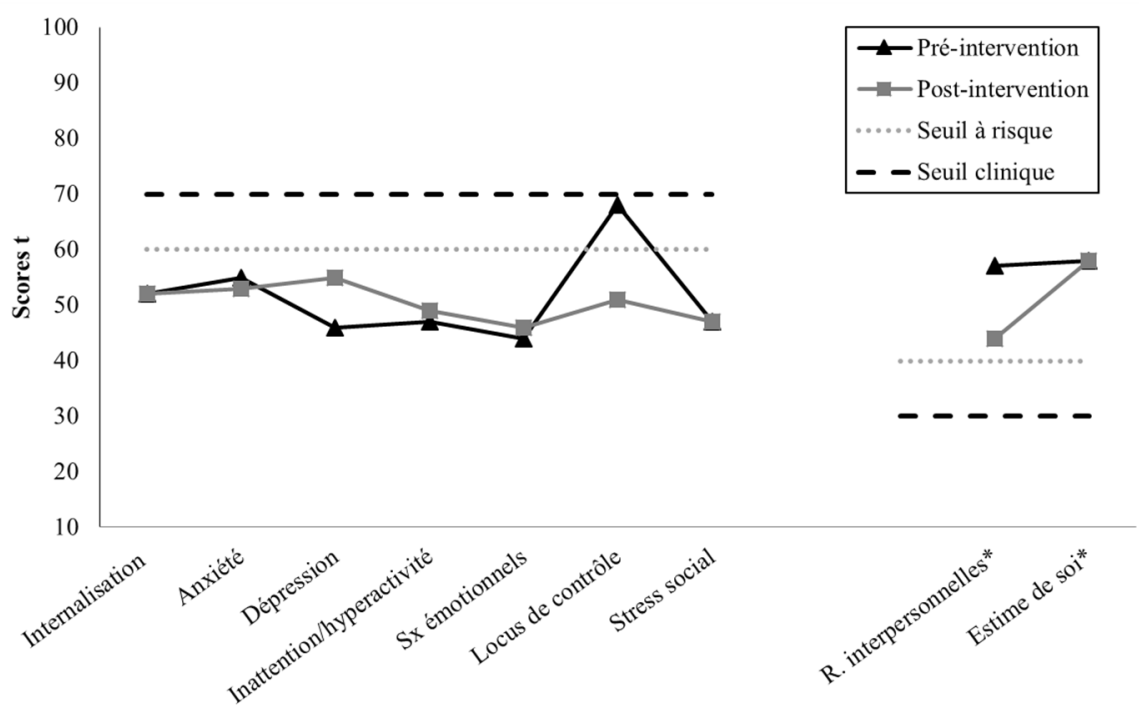

Échelles du BASC-3

Note. Les scores des échelles identifiées avec un astérisque sont renversés

\section{Discussion}

Globalement, les résultats de cette étude pilote sont modestes, mais prometteurs. Les résultats indiquent que pendant la période où ils ont participé au programme Namaste, les deux jeunes étudiés ont connu des améliorations sur plusieurs aspects de leur fonctionnement psychologique. II est possible que la pratique du yoga et les autres activités de l'intervention aient contribué à construire leurs capacités d'autorégulation et à développer des compétences telles que l'autoapaisement et la conscience corporelle dans un cadre sécurisant. Ce programme d'intervention est en effet cohérent avec les recommandations de traitement pour les jeunes ayant un vécu de traumas complexes et qui ont développé des difficultés émotionnelles et comportementales (Cook et al., 2005; Blaustein \& Kinniburgh, 2018). Toutefois, des études plus rigoureuses sur le plan méthodologique s'avèrent nécessaires pour confirmer cette hypothèse.

La négligence, la violence et les ruptures de lien peuvent potentiellement nuire au développement d'un lien positif avec son corps, soi-même et les autres. La participation au programme Namaste avait comme objectif de favoriser chez les jeunes un contact plus sain avec leur monde interne, leurs sensations physiques et leurs émotions, notamment par le biais d'une meilleure autorégulation.

L'autorégulation est un concept large regroupant de nombreuses habiletés utiles dans la vie quotidienne. Les deux jeunes participants ont connu une amélioration de leurs capacités d'autorégulation et une réduction de leurs symptômes d'externalisation et d'internalisation. Des études antérieures sur les interventions basées sur le yoga chez les enfants ou adolescents vont dans le même sens et rapportent des améliorations de l'autorégulation (Butzer et al., 2014; Hariprasad et al., 2013) ou d'autres bénéfices, tels que la réduction de l'activation émotionnelle (Beltran, et al. 2016). Plusieurs écrits ont fait état du potentiel thérapeutique du yoga chez les adultes (Sciarrino et al., 2017), mais peu d'études s'étaient intéressées aux effets de la psychothérapie basée sur le yoga sensible aux traumas sur la santé mentale des enfants et adolescents. Les études de cas rapportées par Spinazzola et ses collaborateurs (2011) illustraient fort bien les impacts cliniques chez des adolescents au vécu similaire, mais l'absence de mesures avant et après l'intervention limitait la portée de leurs conclusions. La présente étude se distingue donc et apporte un premier éclairage sur les effets encore peu documentés de l'utilisation de ce type d'intervention chez une population d'enfants aux multiples problématiques.

Cependant, dans la présente étude, peu d'améliorations ont été observées au niveau des compétences sociales et de l'estime de soi. Ce résultat peut possiblement être expliqué par l'ampleur des difficultés initiales de ces deux jeunes. Un programme de 12 semaines n'est peut-être pas suffisant pour observer des changements sur ces 
construits. Effectivement, la sévérité de la symptomatologie chez les jeunes ayant un vécu de traumas complexes demande souvent un traitement de plus longue durée (Lanktree et al., 2012). Par ailleurs, les éléments liés au soutien des pairs et à l'amélioration des compétences sociales du programme pourraient être bonifiés. Par exemple, Spinazzola et collaborateurs (2011) ont proposé des configurations en quatuor ou en triade pour un meilleur ajustement aux réactions des jeunes et éviter l'attrition.

Cette étude comporte certaines limites qui doivent être soulignées et qui mettent en lumière des pistes de recherche future. D'abord, l'absence d'un groupe contrôle et d'une répartition aléatoire des participants ne permet pas d'attribuer directement les effets positifs observés au programme. De plus, le nombre de cas analysés limite évidemment la généralisation des résultats. Néanmoins, la méthode de la série d'études de cas a permis une analyse détaillée du profil des jeunes et l'illustration des effets spécifiques à chaque individu. II s'agit d'une méthode utile à la compréhension des impacts d'une telle intervention qui a fait l'objet de peu d'études empiriques. La disponibilité de seulement deux temps de mesure est aussi une limite importante de cette étude. L'ajout d'un ou plusieurs temps de mesure avant l'intervention permettrait d'obtenir un niveau de base pour chaque participant et ainsi mieux comparer le seul effet du passage du temps et celui des services déjà en place avec l'effet du programme Namaste. De plus, il est impossible de savoir si les effets positifs observés se sont maintenus dans le temps en raison de l'absence d'un temps de mesure à une période ultérieure (p. ex., six mois ou un an plus tard). Aussi, l'étude actuelle n'évalue pas la fréquence et l'intensité de la pratique du yoga à la maison. Des moyens pour encourager la pratique à la maison pourraient même bonifier le programme et aider les jeunes à intégrer et à généraliser leurs acquis.

L'équipe de Spinazzola (2011) a proposé d'évaluer l'impact de la pratique du yoga sensible aux traumas sur un ensemble de variables comportementales et cliniques telles que les symptômes liés aux traumatismes, l'autorégulation et le contrôle de l'agressivité et de l'impulsivité. Plusieurs des échelles du BASC-3 ont été sélectionnées en ce sens. Cependant, ce questionnaire a ses limites pour évaluer avec précision certains des concepts-clés du programme, tels que la conscience de soi, l'autoapaisement, la sécurité et les frontières personnelles. Puisqu'à notre connaissance un tel outil n'existe pas, il serait essentiel d'en développer un qui soit axé sur ces concepts pour une étude future sur les effets du programme Namaste.

Les répondants aux questionnaires étaient ici des éducateurs qui connaissaient bien les jeunes dans une variété de situations ainsi que les jeunes eux-mêmes. L'utilisation de l'auto-évaluation est importante dans une philosophie d'intervention qui place le jeune au centre de ses préoccupations et qui s'intéresse d'abord à son point de vue. La faible concordance entre les résultats rapportés par les jeunes et leurs éducateurs est probablement due aux difficultés de mentalisation, fréquentes et sévères, chez cette population (Fonagy \& Luyten, 2009).

\section{Conclusion}

Les résultats de ces deux études de cas suggèrent que le programme Namaste peut aider les jeunes qui vivent des difficultés en lien avec leur vécu traumatique. Les observations ici rapportées indiquent plusieurs forces du programme, mais soulèvent aussi certains points à améliorer pour une version future. Des études de plus grandes envergures sont nécessaires pour mieux documenter les effets de cette pratique millénaire comme adjuvant aux autres services offerts pour aider ces jeunes à retrouver un développement plus harmonieux.

\section{Remerciements}

Merci aux jeunes qui ont participé au programme et à leurs familles. Merci à Mathieu Grégoire, Emilie Robert, Ginette Chassé, Samuel Lauzier, Jonathan Laliberté, Valérie Pelletier et Stéphanie Gagnon pour votre confiance et soutien dans ce projet.

\section{Conflit d'intérêt}

Les auteures ne déclarent aucun conflit d'intérêt.

\section{Références}

Bear, M. F., Connors, B. W., \& Paradisio M. A. (2007). Neurosciences: à la découverte du cerveau (3ième édition). Éditions Pradel,

Beltran, M., Brown-Elhillali, A., Held, A., Ryce, P., Ofonedu, M. E., Hoover, D., Ensor, K., \& Belcher, H. M. E. (2016). Yoga-based psychotherapy groups for boys exposed to trauma in urban settings. Alternative Therapies in Health \& Medicine, 22(1), 39-46. 
Beltran, M., Brown-Elhillali, A., Held, A., Deberardinis, C., Muyu, J., Deal, S., \& Haverson, D. (2014). Yoga based psychotherapy: A group protocol for children. The Center for Child and Family Traumatic Stress at Kennedy Krieger Institute.

Butzer, B., Day, D., Potts, A., Ryan, C., Coulombe, S., Davies, B., Weidknecht, B. S., Ebert, M., Flynn, L., \& Khalsa, S. B. S. (2014). Effects of a classroom-based yoga intervention on cortisol and behavior in second and third grade students: A pilot study. Journal of Evidence-based Complementary and Alternative Medicine, 2011), 41-49. https://doi.org/10.1177/2156587214557695

Blaustein, M. E., \& Kinniburgh, K. M. (2018). Treating traumatic stress in children and adolescents: How to foster resilience through attachment, self-regulation and competency. Guilford Press.

Cloitre, M., Stolbach, B. C., Herman, J. L., van der Kolk, B., Pynoos, R., Wang, J., \& Petkova, E. (2009). A developmental approach to complex PTSD: Childhood and adult cumulative trauma as predictors of symptom complexity. Journal of Traumatic Stress, 22(5), 399-408. https://doi.org/10.1002/jts.20444

Collin-Vézina, D., Coleman, K., Milne, L., Sell, J., \& Daigneault, I. (2011). Trauma experiences, maltreatment-related impairments, and resilience among child welfare youth in residential care. International Journal of Mental Health and Addiction, 9(5), 577-589. https://doi.org/10.1007/s11469-011-9323-8

Cook, A., Spinazzola, J., Ford, J., Lanktree, C., Blaustein, M., Cloitre, M., DeRosa, R., Hubbard, R., Kagan, R., Liautaud, J., Mallah, K., Olafson, E., \& van der Kolk, B. (2005). Complex trauma in children and adolescents. Psychiatrics Anals, 35(5), 390-398.

Emerson, D., Sharma, R., Chaudhry, S., \& Turner, J. (2009). Trauma-sensitive yoga: Principles, practice, and research. International Journal of Yoga Therapy, 19(1), 123-128. https://doi.org/10.17761/ijyt.19.1.h6476p8084l22160

Fonagy, P., \& Luyten, P. (2009). A developmental, mentalization-based approach to the understanding and treatment of borderline personality disorder. Development and Psychopathology, 21(4), 1355-1381. https://doi.org/10.1017/S0954579409990198

Galantino, M. L. Galbarry, M., \& Quinn, L. (2008). Therapeutic effects of yoga for children: a systematic review of the literature. Pediatric Physical Therapy, 20(1), 66-80. https://doi.org/10.1097/PEP.0b013e31815f1208

Godbout, N., Girard, M., Milot, T., Collin-Vézina, D., \& Hébert, M. (2018). Répercussions liées aux traumas complexes. In T. Milot, D. Collin-Vézina, \& N. Godbout (Eds.), Le trauma complexe : Comprendre, évaluer et intervenir (pp. 57-90). Presses de l'Université du Québec. https://doi.org/10.2307/j.ctv28683g.7

Hariprasad, V. R., Arasappa, R., Varambally, S., Srinath, S., \& Gangadhar, B. N. (2013). Feasibility and efficacy of yoga as an add-on intervention in attention deficit-hyperactivity disorder: An exploratory study. Indian Journal of Psychiatry, 55(7), 379-384. https://doi.org/10.4103/0019-5545.116317

Kaley-Isley, L. C., Peterson, J., Fischer, C., \& Peterson E. (2010). Yoga as a complementary therapy for children and adolescents: A guide for clinicians. Psychiatry (Edgmont), 7(8), 20-32.

Lanktree, C. B., Brière, J., Godbout, N., Hodges, M., Chen, K., Trimm, L., Adams, B., Maida, C. A., \& Freed, W. (2012). Treating multitraumatized, socially marginalized children: Results of a naturalistic treatment outcomes study. Journal of Aggression, Maltreatment and Trauma, 21(8), 813-828. https://doi.org/10.1080/10926771.2012.722588

Macy, R. J., Jones, E., Graham, L. M., \& Roach, L. (2018). Yoga for trauma and related mental health problems: A meta-review with clinical and service recommendations. Trauma Violence Abuse. 19(1), 35-57. https://doi.org/10.1177/1524838015620834

Mayer, S. (2019). Enhancing the lives of children in out-of-home care: An exploration of mind-body interventions as a method of trauma recovery. Journal of Child and Adolescent Trauma, 12(4), 549-560. https://doi.org/10.1007/s40653-019-0250-3

Milot, T., Collin-Vézina, D., \& Godbout, N. (2018). Qu'est-ce que le trauma complexe? In T. Milot, D. Collin-Vézina, \& N. Godbout (Eds.), Le trauma complexe: Comprendre, évaluer et intervenir (pp. 9-36). Presses de l'Université du Québec. https://doi.org/10.2307/j.ctv28683g.5

Nolan, C. R. (2016). Bending without breaking: A narrative review of trauma-sensitive yoga for women with PTSD. Complementary Therapeutic Clinical Practice, 24, 32-40. https://doi.org/10.1016/j.ctcp.2016.05.006

Price, M., Spinazzola, J., Musicaro, R., Turner, J., Suvak, M., Emerson, D., \& van der Kolk, B. (2017). Effectiveness of an extended yoga treatment for women with chronic post-traumatic stress disorder. Journal of Alternative and Complementary Medicine, 23(4), 300-309. https://doi.org/10.1089/acm.2015.0266

Reynolds, C. R., \& Kamphaus, R. W. (2015). BASC-3 Système d'évaluation du comportement de l'enfant (3e éd.), Version pour francophones du Canada. NCS, Pearson Inc.

Reynolds, C. R., \& Kamphaus, R. W. (2016). Behavior assessment system for children, 3rd edition. Assessment services: Publication data. NCS, Pearson Inc.

Ross, A., \& Thomas, S. (2010). The health benefits of yoga and exercise: A review of comparison studies. Journal of Alternative and Complementary Medicine, 16(1), 3-12. https://doi.org/10.1089/acm.2009.0044 
Taylor, J., McLean, L., Korner, A., Stratton, E., \& Glozier, N. (2020). Mindfulness and yoga for psychological trauma: systematic review and meta-analysis. Journal of Trauma and Dissociation, 1-38. Advance online publication. https://doi.org/10.1080/15299732.2020.1760167

Sciarrino, N. A., DeLucia C., O'Brien, K., \& McAdams, K. J. (2017). Assessing the effectiveness of yoga as a complementary and alternative treatment for post-traumatic stress disorder: A review and synthesis. Alternative Complement Medecine, 23(10), 747-755. https://doi.org/10.1089/acm.2017.0036

Spinazzola, J., Rhodes, A. M., \& Emerson, D. (2011). Application of yoga in residential treatment of traumatized youth. Journal of the American Psychiatric Nurses Association, 1766), 431-444. https://doi.org/10.1177/1078390311418359

van der Kolk, B. (2014). The body keeps the score: brain, mind, and body in the healing of trauma. Viking Penguin. 